\title{
Method for Measuring the Dielectric Constant of Ferroelectric Ceramics at S-Band Frequencies
}

\author{
by C. B. SHARPE and C. G. BROCKUS
}

Electronic Defense Group, Deparfment of Electrical Engineering, The University of Michigan, Ann Arbor, Michigan

\begin{abstract}
A method is presented for measuring the complex dielectric constant of ferroelectric ceramics at microwave frequencies. The method employs a disk-shaped sample in a radial-line configuration at the end of a coaxial-line structure. An equivalent circuit, established by admittance measurements, is used to relate the radial admittance at the sample face to the measured admittance on the slotted line preceding the coaxial sample holder. The nonuniform variation of electric field over the face of the sample is taken into account by means of a continued fraction expansion of $x J_{1}(x) / J_{0}(x)$ in terms of $x=k^{\prime} a$, where $k^{\prime}$ is the wave number in the ferroelectric sample and $a$ is its radius. Representative data on the variation of the complex dielectric constant with temperature at $3 \mathrm{kmc}$. are given for two samples of $\mathrm{BaTiO}_{3}$ fired under atmospheric pressure and $5000 \mathrm{lb}$. per sq. in. The frequency spectrum of hot-pressed $\mathrm{Cd}_{2} \mathrm{Nb}_{2} \mathrm{O}_{7}$ in the range 1.8 to $4.0 \mathrm{kmc}$. is also presented.
\end{abstract}

\section{Introduction}

B ECAUSE of their very high dielectric constants, ferroelectrics present special measurement problems at microwave frequencies. These problems stem largely from the contraction of wave length in the material, which gives rise to nonuniform field effects and makes the measurement particularly sensitive to the shape and orientation of the sample in the wave guide or cavity. As a result, previous workers have found it necessary to modify conventional techniques, by using quarter-wave matching sections, for example, or to employ them in combination for frequencies above $1 \mathrm{kmc}^{1}$ The purpose of this paper is to present a method based on the ordinary impedance technique which has been used by the writers to measure a variety of ferroelectric ceramics in the frequency range 1.8 to $4.0 \mathrm{kmc}^{2}$ The method

Received October 13, 1959; revised copy received January 28, 1960 .

This work was sponsored by the U. S. Army Signal Research and Development Laboratory. Fort Monmouth, N. J., under Contract No. DA-36-039 sc-75003.

The writers are, respectively, associate professor and research assistant, Electronic Defense Group, Department of Electrical Engineering, The University of Michigan.

1 (a) J. G. Powles and W. Jackson, "Measurement of Dielectric Properties of High-Permittivity Materials at Centimeter Wave Lengths," Proc. Inst. Elec. Engrs. (London), Part III, 96, 383-89 (1949).

(b) A. von Hippel, "Ferroelectricity, Domain Structure, and Phase Transitions of Barium Titanate," Revs. Modern Phys., 22 [3] 221-37 (1950).

(c) L. Davis, Jr., and L. G. Rubin, "Some Dielectric Properties of Barium-Strontium Titanate Ceramics at 3000 Megacycles," J. Appl. Phys., 24 [9] 1194-97 (1953); Ceram. Abstr., 1954, January, p. 11g.

2 C. B. Sharpe and C. G. Brockus, "Investigation of Microwave Properties of Ferroelectric Materials," University of Michigan, Electronic Defense Group, Final Report on Contract No. DA-36039 sc-75003; March 1959.
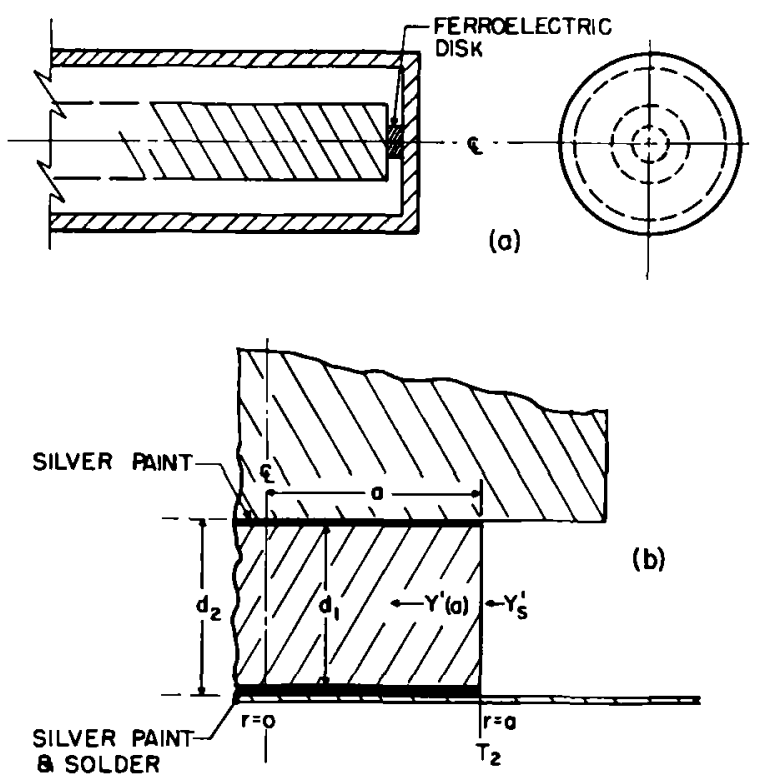

Fig. 1. (a) Simplifled drawing of sample holder and (b) detail of somple.

is suitable for the measurement of the complex dielectric constant of ferroelectrics with respect to variations in both temperature and bias field, and the samples are easily fabricated.

\section{Theory}

The coaxial-line structure and disk-shaped sample are illustrated in Fig. 1. At frequencies below several hundred megacycles per second the sample can be represented to a good approximation by a lossy capacitor terminating a uniform line. In this case one can readily determine the relative dielectric constant, $\epsilon / \epsilon_{0}=\epsilon^{\prime}-j \epsilon^{\prime \prime}$, knowing the impedance at the capacitor terminals. At higher frequencies this procedure breaks down in several respects. It becomes impossible to identify the terminals of the effective capacitance, and the fringing fields are no longer negligible. Also, the measured capacity of the sample cannot be simply related to the dielectric constant because of the nonuniform variation of the electric field throughout the sample.

These difficulties were overcome by considering the sample disk as a section of radial line ${ }^{3}$ as suggested by the expanded view of the sample in Fig. 1(b). Only the lowest radial-line mode will be excited in the sample if the height of the radial line, $d_{2}$, is small compared with the wave length $\lambda$ in air.

${ }^{3}$ Principles of Microwave Circuits, Massachusetts Institute of Technology Radiation Laboratory Series, Vol. 8, Chapter 8: C. G. Montgomery, R. H. Dicke, E. M. Purcell, editors. McGrawHill Book Co., Inc., New York, 1948.486 pp. 


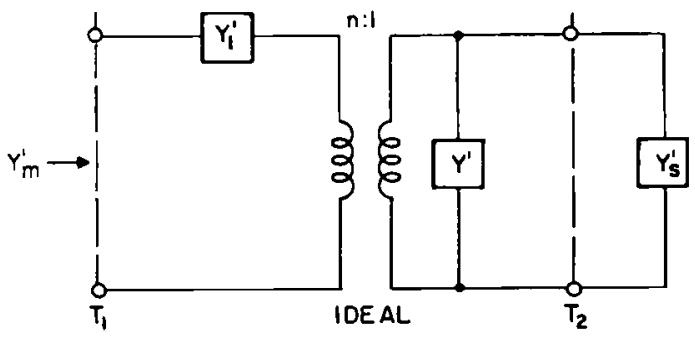

Fig. 2. Equivalent circuit for sample holder.

The thick center conductor permits the higher-order modes generated near the corner to attenuate before reaching the sample. This allows one to establish a reference plane $T_{2}$ for the equivalent circuit at the sample-air interface as shown in the figure. The normalized admittance $Y^{\prime}$, looking into the sample at $T_{2}$ can be calculated in terms of the dielectric constant of the sample by radial-line theory. The remaining problem is to relate $Y^{\prime}$, to the measured admittance $Y^{\prime}{ }_{m}$ at some chosen reference plane $T_{1}$ in the slotted line preceding the sample holder. This is done by noting that at any two reference planes where only the dominant mode propagates, any two-port lossless junction can be represented by the equivalent circuit shown in Fig. 2 , where $Y^{\prime}{ }_{1}$ and $Y^{\prime}$ are normalized susceptances and $n$ is real. ${ }^{4}$ If $T_{1}$ is located at a null when the sample is replaced by a metal disk of the same height and radius as the sample, then $Y_{1}^{\prime}=\infty$. The remaining elements of the equivalent circuit must be determined at each frequency. This is most easily done by replacing the sample with two known dielectrics. Rutile $\left(\mathrm{TiO}_{2}\right)$ and air have proved to be satisfactory for this purpose. In the case of rutile it is assumed that there is negligible dispersion in the dielectric constant from $250 \mathrm{mc}$., where the measurement is made, to $4 \mathrm{kmc}$. Then

$$
Y^{\prime}{ }_{s}=n^{2} Y_{m}^{\prime}-Y^{\prime}
$$

where the admittance $Y^{\prime}{ }_{m}$ measured in the slotted line is

$$
Y_{m}^{\prime}=\frac{\rho\left(1+\tan ^{2} \frac{2 \pi \Delta}{\lambda}\right)-j\left(\rho^{2}-1\right) \tan \frac{2 \pi \Delta}{\lambda}}{1+\rho^{2} \tan ^{2} \frac{2 \pi \Delta}{\lambda}}
$$

and $\rho$ is the measured voltage standing-wave ratio when the line is terminated in the sample. The distance $\Delta$ measures the shift in the standing wave toward the generator when the sample is replaced by the metal short.

It should be noted that the equivalent circuit accounts for not only the fringing fields in the sample holder but also for all other discontinuities in the line between the reference planes, including the connectors and the tapered section.

Assuming the electric field $E_{z}$ is a maximum at the center of the sample, the normalized input admittance $Y^{\prime}(a)$ at $T_{2}$ just inside the sample, looking in the direction of decreasing radius, can be shown to be*

$$
Y^{\prime}(a)=j \frac{J_{1}\left(k^{\prime} a\right)}{J_{0}\left(k^{\prime} a\right)}
$$

where

$$
k^{\prime}=(2 \pi / \lambda) \sqrt{\epsilon^{\prime}-j \epsilon^{\prime \prime}}
$$

is the wave number in the sample. The boundary condition to be satisfied at the air-dielectric interface is $Y(a)=Y_{s}$, where $Y(a)$ is the admittance in the dielectric at $r=a$ and
$Y$, is the admittance in air at $r=a$. In general the admittance $Y(r)$ at any radius $r$ is related to the normalized admittance $V^{\prime}(r)$ by

$$
Y(r)=Y_{0}(r) Y^{\prime}(r)
$$

where

$$
Y_{0}(r)=\frac{2 \pi r}{d} \sqrt{\frac{\epsilon}{\mu_{0}}}
$$

is the characteristic admittance of the radial line and $d$ its height. Referring to Fig. 1(b), the height of the line within the sample, $d_{1}$, differs from that outside the sample, $d_{2}$, owing to the thickness of silver plating and solder which is applied in mounting the sample. In the sample holder to be described, $d_{1}=0.019$ in. and $d_{2}=0.0205$ in. It follows that

$$
Y^{\prime}(a)=\frac{d_{1}}{d_{2}} \frac{1}{\sqrt{\epsilon^{\prime}}-j \epsilon^{\prime \prime}} Y^{\prime \prime} \text {, }
$$

Using equations (3) and (4) this may be written at $x=k^{\prime} a$ as

$$
w=x \frac{J_{1}(x)}{J_{0}(x)}=-j \frac{d_{1}}{d_{2}}\left(\frac{2 \pi a}{\lambda}\right) Y^{\prime}
$$

where $w$ is known and $x$ is to be determined. The solution of equation (8) can be accomplished algebraically to any desired degree of approximation by noting that $w$ can be represented by the continued fraction 5

$$
w=\frac{2(x / 2)^{2}}{1-\frac{(x / 2)^{2}}{2-\frac{(x / 2)^{2}}{3-(x / 2)^{2}}}}
$$

Terminating equation (9) with the terms shown leads to a quadratic in $x^{2}$, from which the desired dielectric constant is obtained:

$$
\begin{gathered}
\epsilon^{\prime}-j \epsilon^{\prime \prime}=x^{2}\left(\frac{\lambda}{2 \pi a}\right)^{2} \\
=\left(\frac{\lambda}{\pi a}\right)^{2}\left[\frac{9 w+24-\sqrt{9(3 w+8)^{2}-24 w(w+12)}}{w+12}\right]
\end{gathered}
$$

This approximation is good to within $1 \%$ for real $x$ less than 2.2 . This restriction on the upper value of $x=k^{\prime} a$ places an upper frequency limit of about $5 \mathrm{kmc}$. on the impedance method for dielectric constants in the order of 4000 , depending, of course, on the precision with which the samples can be fabricated. In principle, one can operate at a frequency above the first resonance in $Y_{\text {, }}$, which occurs at $x=2.405$, and employ a Taylor expansion of $w$ in the region of operation. This results, however, in a significant loss of accuracy in the determination of $\epsilon^{\prime}$ due to the relatively high loss tangent of the ferroelectrics in the microwave range and to the pronounced mismatch at the sample face.

\section{Experimental Equipment and Procedure}

Figure 3 is an assembly drawing of the sample holder. The purpose of the tapered section is to minimize the effect

\footnotetext{
4 Waveguide Handbook, Massachusetts Institute of Technology Radiation Laboratory Series, Vol. 10, p. 119; N. Marcuvitz, editor. McGraw-Hill Book Co., Inc., New York, 1951. 428 pp.

See p. 265 of footnote 3.

5 E. T. Whittaker and G. N. Watson, Modern Analysis, p. 380. Cambridge University Press, London, 1946. 608 pp.
} 


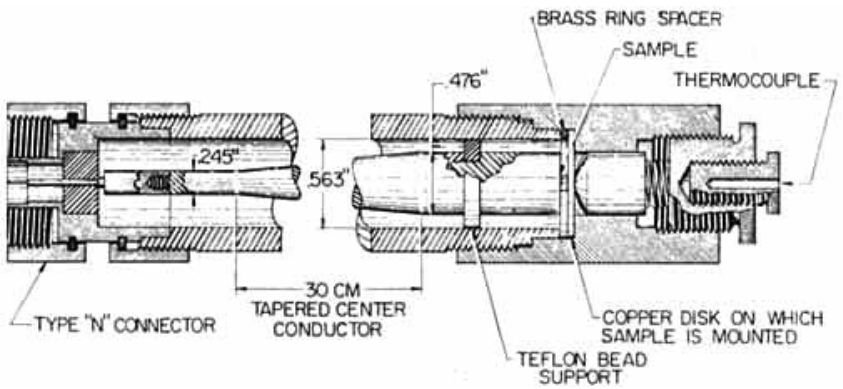

Fig. 3. Sample holder assembly.

of position errors in the measurement of $Y^{\prime}{ }_{m}$. It is shown in the Appendix (see p. 305) that for a given deviation in the measurement of the standing-wave minimum, the least error in the measured susceptance occurs when that susceptance is equal to the characteristic admittance of the slotted line. Since the 50-ohm impedance of the slotted line used in this experiment is substantially higher than the measured reactance of the sample for most ferroelectrics in the S-band range, a transmission-line taper was employed (in the manner of an ideal transformer) to obtain a more nearly optimum load admittance in the slotted line. The taper shown in Fig. 3 was designed after the method of Willis and Sinha ${ }^{6}$ to provide a five-to-one impedance transformation, which represents a reasonable compromise between the foregoing electrical considerations and the physical limitation of taper length.

The disk-shaped samples are fabricated in the following manner: Using a diamond wheel, a ceramic sheet is first cut from the rough sample stock to the approximate thickness. After surface grinding to the $d_{1}(0.019$ in.) dimension, this sheet is painted on both sides with Du Pont No. 4731 silver paint, fired at $600^{\circ} \mathrm{C}$. for 22 minutes, and cooled in the furnace. The painted sheet is then resurfaced to a combined electrode thickness of $0.001 \mathrm{in}$. The individual samples are cut from this sheet with an ultrasonic cutter and then carefully centered and soldered on a thin (0.005-in.) flexible copper disk. This disk supports the sample in the holder, and the spring-loaded plug pressing against its back in the assembled sample holder assures contact between the sample and the center conductor.

Figure 4 is a schematic diagram of the experimental setup. Two slotted lines (Hewlett-Packard 805A) were employed, one for making the admittance measurements of $Y^{\prime}{ }_{m}$ and the other for resetting the frequency of the signal generator. The latter was required because the parameters of the equivalent circuit are quite sensitive to deviations in frequency. Both lines were modified with a 40-threads-per-in. lead-screw drive on the carriage and fitted with dial gauges having a $15-\mathrm{cm}$. range with graduations of $0.01 \mathrm{~mm}$. The voltage standingwave ratios were measured using the method of Winzemer. ${ }^{7}$ This method does not require a calibrated attenuator and gives results independent of the detector characteristics. The bias voltage indicated in the figure was applied through the side arm of a coaxial $T$ junction. Isolation from the traveling-wave amplifier was provided by a General Radio coupling capacitor.

6 J. Willis and N. K. Sinha, "Non-Uniform Transmission Lines as Impedance Transformers," J. Inst. Elec. Engrs. (London), Part $V I I B, 103,166-72(1956)$

T A. M. Winzemer, "Methods for Obtaining the Voltage Standing-Wave Ratio on Transmission Lines Independently of the Detector Characteristics," Proc. I.R.E. (Inst. Radio Engrs.), 38, 275-79 (March 1950).

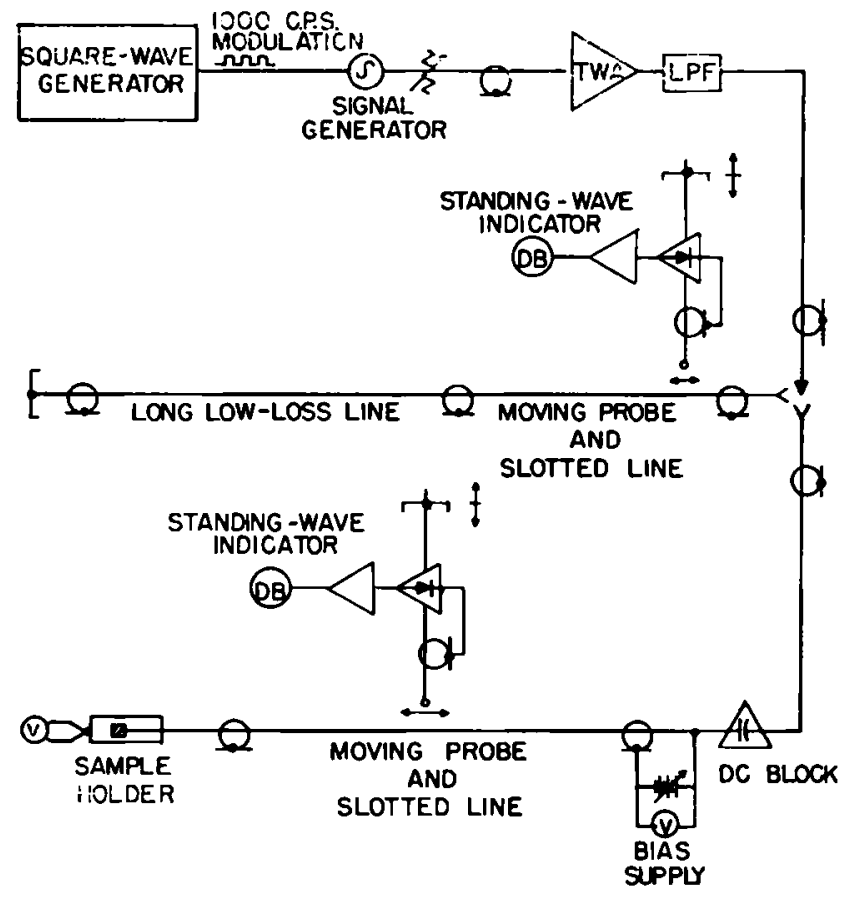

Fig. 4. Experimental arrangement for measurement of the complex dielectric constant of ferroelectrics.

\section{Results}

In the report ${ }^{2}$ referred to previously the writers have presented the results of dielectric constant measurements on a number of ferroelectric ceramics. Representative examples of these measurements are presented in Figs. 5 and 6 . The upper graph in Fig. 5 shows the variation of the complex dielectric constant with temperature at $3 \mathrm{kmc}$. for ceramic barium titanate pressed at $5000 \mathrm{lb}$. per sq. in. and fired in the conventional manner. In the lower graph, similar curves are shown for hot-pressed barium titanate. This ceramic was fired at 130()$^{\circ} \mathrm{C}$. for $1 / 2$ hour under a pressure of 5000 lb. per sq. in. and for this reason is particularly dense. The effect of this increase in density and the resulting reduction in grain size is apparent from the figures.

Figure 6 presents the dielectric constant of hot-pressed cadmium niobate versus frequency in the range 1.8 to $4.0 \mathrm{kmc}$. The absence of any relaxation in this range is associated with the low Curie temperature of this ferroelectric $\left(185^{\circ} \mathrm{K}\right.$.). It was also observed that at room temperature there is no appreciable dependence of the dielectric constant on electric field. The relation between the Curie temperature and the frequency and field dependence of the dielectric constant for perovskite-like ferroelectrics has been established on theoretical grounds by Diamond. ${ }^{8}$

8 (a) Howard Diamond, "Theory of Electric Field Nonlinearity in the Perovskite-Like Ferroelectrics"; presented at the Fall Meeting, Electronics Division. The American Ceramic Society, Detroit, Mich., Sept. 25, 1959 (No. 7-L-59F).

(b) H. Diamond, "On the Polarization, Microwave Dispersion, and Loss in High-Permittivity Ferroelectrics," Willow Run Laboratories Rept. No. 2900-121-T, University of Michigan. Ann Arbor; January 1960. 

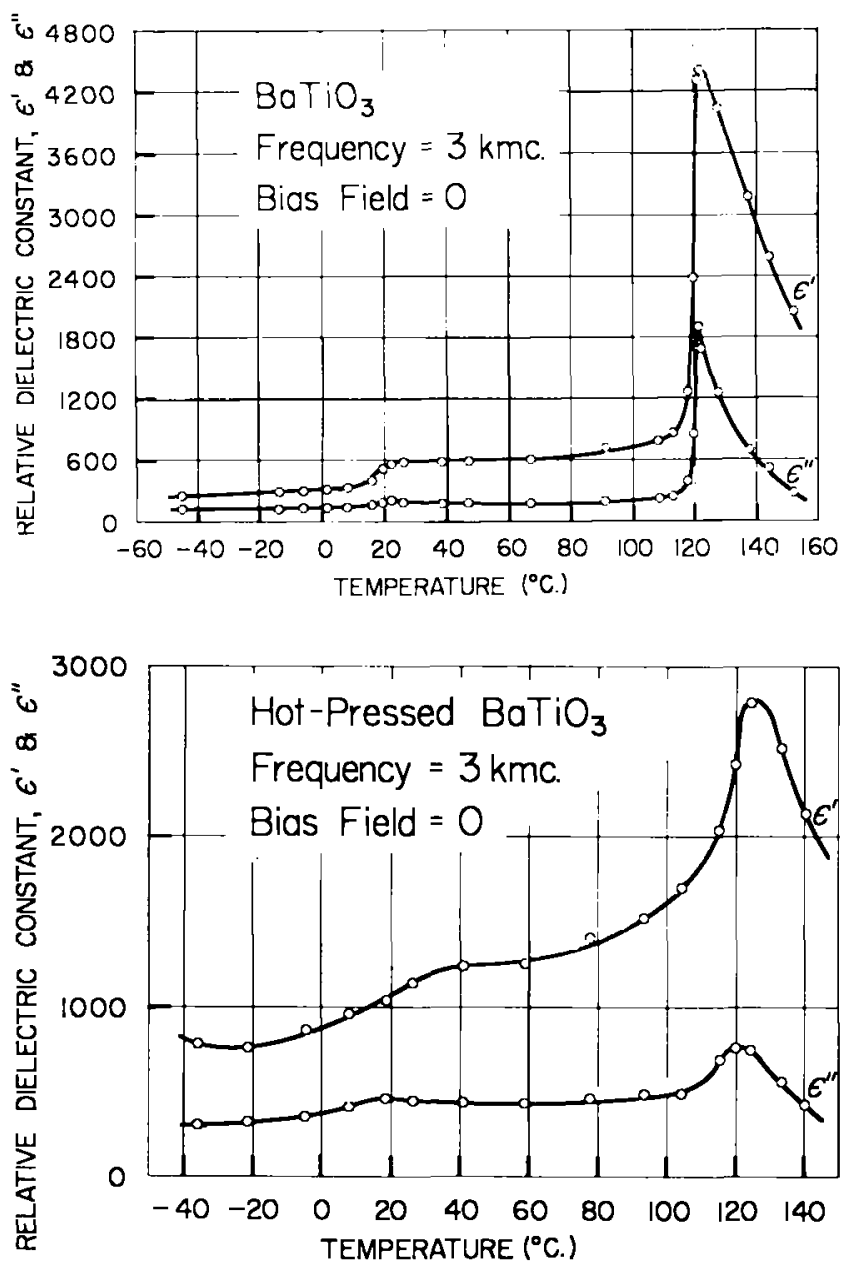

Fig. 5. Complex dielectric constant vs. temperature for ceramic $\mathrm{BaTiO}_{3}$ fred under different pressure condifions.

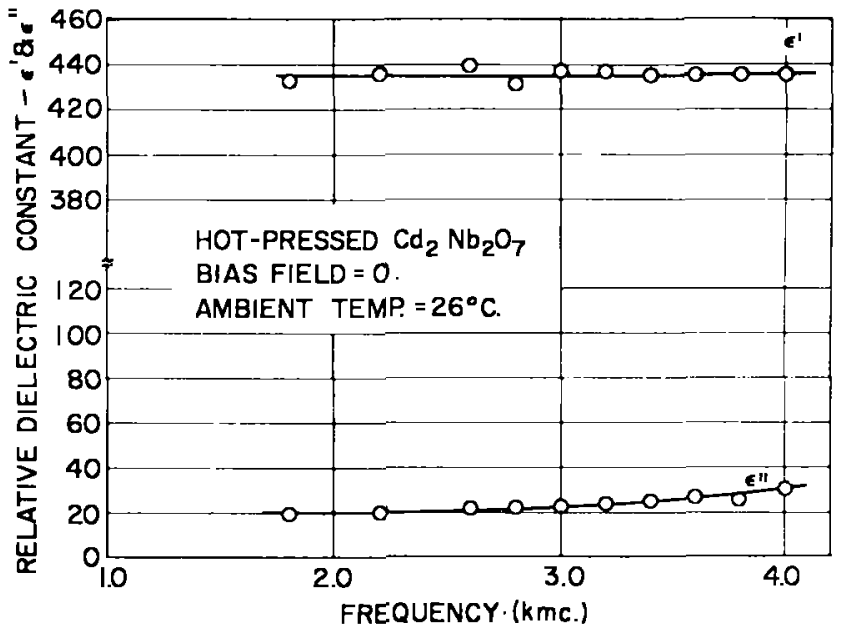

Fig. 6. Complex dielectric constant vs. frequency for hot-pressed $\mathrm{Cd}_{2} \mathrm{Nb}_{2} \mathrm{O}_{7}$.

and taking the derivative with respect to $Y^{\prime}{ }_{m}$, while holding $\rho$ constant. There results

$$
d \theta=K \frac{d Y_{m}^{\prime}}{Y_{m}^{\prime}}
$$

where the sensitivity is defined by

$$
K=\frac{j Y_{m}^{\prime}}{Y_{m}^{\prime}{ }_{m}^{2}-1}
$$

One now wishes to determine the value of $Y^{\prime}{ }_{m}$ which maximizes $|K|$, assuming $\rho$ is held constant. This is most easily done by expressing $|K|^{2}$ in terms of the measured reflection coefficient, $\Gamma_{m}=\gamma e^{j \psi}$, which can be defined by the relation

$$
Y_{m}^{\prime}=\frac{1-\Gamma_{m}}{1+\Gamma_{m}}
$$

It follows that

$$
|K|^{2}=\frac{\gamma^{4}+1-2 \gamma^{2} \cos 2 \psi}{16 \gamma^{2}}
$$

Since, from

$$
\gamma=\frac{p-1}{p+1}
$$

The writers are indebted to $\mathrm{H}$. Diamond for his advice and as-
sistance in the early phases of the work and for his aid in selecting the ferroelectrics which were investigated. A special acknowl. edgment is due V. Leipa, who made the measurements. The writers owe thanks also to $V$. Chang, who prepared the ceramics, and to $\mathrm{H}$. Senecal, who developed the fabrication technique.

The hot-pressed ceramics were kindly supplied by $A$. de Bretteville of the U. S. Army Signal Research and Development Laboratory. The hot-pressing technique was devised by the Inorganic Dielectric Section, Materials Branch, U. S. Army

Signal Research and Development Laboratory.

\section{APPENDIX}

The condition which results in the least error (percentagewise) in measured admittance $Y^{\prime}{ }_{m}$ for a given deviation in the position of the standing-wave minimum is identical to that which makes the measured angular shift in the standing wave, $\theta=2 \pi \Delta / \lambda$, most sensitive to a relative change in $Y_{m}^{\prime}$. This sensitivity can be calculated for a specified standing-wave ratio by rewriting equation (2) as

$$
\theta=\tan ^{-1}\left[j\left(\frac{Y_{m}^{\prime}-\rho}{\rho \bar{Y}_{m}^{\prime}-1}\right)\right]
$$

$\gamma$ is a constant if $\rho$ is held constant, the maximum value of $|K|$ occurs when $\psi= \pm \pi / 2$. Taking the lower sign appropriate for a capacitive load, the optimum normalized admittance is

$$
Y_{m}^{\prime}=G_{m}^{\prime}+j B_{m}^{\prime}=\frac{1+j \gamma}{1-j \gamma}
$$

In terms of $\rho$,

$$
G_{m}^{\prime}=\frac{2 \rho}{\rho^{2}+1} ; B_{m}^{\prime}=\frac{\rho^{2}-1}{\rho^{2}+1}
$$

If $\rho$ is also allowed to vary, it can be seen from equation (I-5) that $|K|$ assumes a largest maximum value of $|K|=1 / 2$ when $\gamma=1(\rho=\infty)$ and $\psi= \pm \pi / 2$. The corresponding values of $G^{\prime}{ }_{m}$ and $B^{\prime}{ }_{m}$ are

$$
G_{m}^{\prime}=0 ; B^{\prime}{ }_{m}=1
$$

Thus, when the measured admittance is purely susceptive, the optimum choice of line admittance is the value $Y_{0}=B_{m}$. When $Y^{\prime}{ }_{m}$ contains a conductive term, the general condition given by equation (I-8) differs from equation (I-9) to a negligible extent if $1<<\rho^{2}$. 